Article

\title{
Screening, Identification, and Optimization of Fermentation Conditions of an Antagonistic Endophyte to Wheat Head Blight
}

\author{
Peigen Zhang ${ }^{1, \dagger}$, Yongxing Zhu ${ }^{1,+}$, Dongfang Ma ${ }^{1,2}$, Wenjie Xu ${ }^{1}$, Jingjing Zhou ${ }^{1}$, \\ Hanwen Yan ${ }^{1}$, Lei Yang ${ }^{1}$ and Junliang Yin ${ }^{1, *}$ \\ 1 Hubei Key Laboratory of Waterlogging Disaster and Agricultural Use, College of Agriculture, \\ Yangtze University, Jingzhou 434025, China \\ 2 State Key Laboratory of Biology of Plant Diseases and Insects, Institute of Protection, Beijing 100193, China \\ * Correspondence: yinjunliang@yangtzeu.edu.cn \\ $\dagger$ These authors contributed equally to this work.
}

Received: 21 July 2019; Accepted: 21 August 2019; Published: 22 August 2019

\begin{abstract}
Fusarium Head Blight (FHB, scab) is a destructive fungal disease that causes extensive yield and quality losses in wheat and other small cereals. Biological control of FHB is considered to be an alternative disease management strategy that is environmentally benign, durable, and compatible with other control measures. In this study, to screen antagonistic bacteria with the potential to manage FHB, 113 endophytes were isolated from the stems, leaves, panicles, and roots of wheat. Among them, six strains appeared to effectively inhibit Fusarium graminearum growth and one isolate, XS-2, showed a highly antagonistic effect against FHB. an in vitro antagonistic test of XS-2 on wheat heads confirmed that XS-2 could suppress the disease severity of FHB. The $16 \mathrm{~S}$ rDNA sequence analysis revealed that XS-2 is a strain of Bacillus amyloliquefaciens. Antagonistic spectrum analyses showed that XS-2 had antagonistic effects against two and four types of cotton and fruit tree pathogens, respectively. The fermentation condition assays showed that glucose and peptone are the most suitable nutrient sources for XS-2, and that the optimal $\mathrm{pH}$ value and temperature for fermentation were 7.4 and $28^{\circ} \mathrm{C}$, respectively. Our study indicates that XS-2 has a good antagonistic effect on FHB and lays a theoretical foundation for the application of the strain as a biological agent in the field to control FHB.
\end{abstract}

Keywords: Fusarium graminearum; endophyte bacterium; $16 \mathrm{~S}$ rDNA; Bacillus amyloliquefaciens; biocontrol agent

\section{Introduction}

Fusarium head blight (FHB), which is mainly caused by Fusarium graminearum, is one of the most serious diseases of wheat and other small grain cereals in both temperate and semi-tropical regions [1]. It can cause the whole plant to rot, including seeds, seedlings, stalks, stems, and ears, and yield loss can reach up to $70 \%$ in some crop fields [2]. Besides the economic losses caused by reductions in the grain yield, another main problem is potential contamination of wheat seeds with mycotoxins, mainly deoxynivalenol (DON), which poses a serious threat to food safety and animal health [3]. Although there are cultivars that are moderately resistant to FHB, such as 'Sheng selected No. 6', these are usually susceptible to powdery mildew and stripe rust, which makes it difficult to deploy these FHB-resistant varieties in the field [4].

Presently, the control of FHB is highly dependent on the application of agrochemicals [5]. However, the chemical control method also results in many problems [6]. First, FHB mostly occurs when wheat plants are flowering, which overlaps with the rainy period of the summer season. The pesticides 
are easily washed away by rainwater, which limits the control efficiency. Second, there is growing concern about the negative effects of chemical pesticides, particularly about their potentially toxic effects on humans and animals. Thus, alternative disease management strategies are needed to fulfill the consumer demand for pesticide-free food while maintaining environmental safety.

Endophytes, such as bacterial and fungi microorganisms, are widely distributed in all living plants. They do not directly cause any plant diseases, but some antagonize the infection, colonization, and development of other plant pathogens [7]. a large number of studies have shown that endophytic bacteria are correlated with enhanced plant growth under both abiotic (heat, drought, salinity, heavy metal exposure, etc.) and biotic stresses, including Fusarium head blight [8-10]. For example, Comby et al. [8] isolated 86 microorganisms from wheat plants to find endophytes with the potential to become biological control agents against $\mathrm{FHB}$, and 13 strains conferred significant protection against FHB on wheat spikelets. Dal Bello et al. [11] found that a strain of Trichoderma harzianum could inhibit Fusarium head blight by a rate of $79 \%$. Combined effects of different kinds of endophyte and other materials, such as $\mathrm{ZnO}$ nanoparticles, have also been reported. For example, mixtures of multiple isolates of Pseudomonas, Erwinia, and Bacillus spp. have been reported to exert greater antagonistic effects against the growth of F. graminearum than a single isolate [12]. Dimkpa et al. [13] and Palazzini et al. [14] demonstrated that $\mathrm{ZnO}$ nanoparticles combined with biocontrol bacteria (Pseudomonas chlororaphis O6) could significantly inhibit the growth of F. graminearum in wheat. These studies indicate that endophytes have a significant potential to control FHB.

Currently, limited useful biocontrol resources are available to manage FHB. Thus, there is an urgent need to identify more endophytic resources and to evaluate their antagonistic abilities against FHB. In this study, we aimed to explore biocontrol resources for FHB. In this context, our objectives were: (1) to isolate endophytic bacteria from wheat tissues and determine their antagonistic effects on F. graminearum, (2) to classify the endophyte strain XS-2, which was isolated from wheat spikelets and showed the greatest antagonistic effect on F. graminearum, using $16 \mathrm{~S}$ rDNA sequence analysis, (3) to preliminarily study the strain's suitable nutrient sources and optimal growth conditions to provide a theoretical basis for the usage of XS-2 as a biological control agent, and (4) to explore the antagonistic effect of XS-2 on FHB in wheat heads in vitro.

\section{Materials and Methods}

\subsection{Plant Sample Collection and Endophyte Isolation}

Wheat samples were collected from farmland near the west campus of Yangtze University in Jingzhou, Hubei province, China (N30 $21^{\prime} 32.53^{\prime \prime}$ E112 $\left.08^{\prime} 22.05^{\prime \prime}\right)$. Samples were collected in an aseptic bag and were taken back to the laboratory immediately. Wheat stems, leaves, roots, and spikelets were rinsed with clean water and cut into 5 -cm segments. Segments were then soaked in $75 \%$ alcohol for $30 \mathrm{~s}$, rinsed once in sterile water, soaked for $5 \mathrm{~min}$ in $1 \%$ mercury chloride, and rinsed three times with sterile water. Using a sterile mortar, the sample was grinded with $5-\mathrm{mL}$ of sterile water, and $100 \mu \mathrm{L}$ of the solution was added to Potato Dextrose Agar (PDA: $20 \mathrm{~g}$ of dried potato infusion, $20 \mathrm{~g}$ of glucose, $15 \mathrm{~g}$ of agar per L) and to Nutrient Agar (NA: $10 \mathrm{~g}$ of peptone, $3 \mathrm{~g}$ of beef extract, $2.5 \mathrm{~g}$ of glucose, $18 \mathrm{~g}$ of agar per L) media, respectively. Solutions were spread on plates uniformly with a spreader, before being incubated at $28{ }^{\circ} \mathrm{C}$ for 2 to 3 days. Emergent colonies were picked and transferred onto PDA or NA fresh media, respectively, to obtain pure culture isolations.

\subsection{Dual Culture Assays for Antagonistic Tests}

Dual culture assays were used to test the antagonistic effects of isolations on Fusarium graminearum [8]. For each repetition, a 5-mm mycelium pellet of $F$. graminearum was placed onto the center of an NA plate and the endophyte was placed between the F. graminearum pellet and the plate edge. The plates with $F$. graminearum growths alone were used as controls. Then, dual culture and control plates were incubated at $28{ }^{\circ} \mathrm{C}$ in the dark until the F. graminearum fully 
covered the control plate. The inhibition zone was measured and statistically analyzed. To measure the inhibition zone, lines were drawn from the plate center to the edge, and the longest line segment overlapping the endophyte growth area was measured and recorded as the dimeter of the inhibition zone. Each endophyte/pathogen combination was duplicated at least five times and two independent experimental repetitions were done for each strain.

The same method was used to detect the inhibitory effects of antagonistic endophyte on other common plant pathogens, including Fusarium moniliforme and Rhizoctonia solani (two cotton pathogens), Sclerotium rolfsii (a rice pathogen), Alternaria alternata (a pear fruit pathogen), Colletotrichum truncatum (a pitaya fruit pathogen), and Colletotrichum gloeosporioides (an apple fruit pathogen). Each treatment included three repetitions.

\subsection{Classification of Endophyte Isolates}

A modified version of the CTAB (cetyltriethylammnonium bromide) method was used to extract the DNA of the endophyte isolates [15]. Additionally, the extracted DNA was used as template for polymerase chain reaction (PCR) amplification. The 16S rDNA universal primers (27F: 5'-CAGAGTTTGATCCTGGCT-3', 1492R: 5'-AGGAGGTGATCCAGCCGCA-3') were used to amplify the target fragments. In addition, the amplified products were sequenced by Sangon Biotech (Shanghai, China). Obtained sequences were used to perform BLAST searching on the NCBI website (http://www.ncbi.nlm.nih.gov/) to determine the possible species of the isolates. BLAST results were downloaded and the sequences were used to construct the phylogenetic tree using MEGA7.0 [16].

\subsection{Optimization of Fermentation Conditions}

For growth curve determination, the endophyte isolate was cultured in NA liquid medium and incubated in the dark at $28^{\circ} \mathrm{C}$ with shaking at $180 \mathrm{rpm}$ (pH 7.0). The $\mathrm{OD}_{600}$ value was measured every $4 \mathrm{~h}$ until $36 \mathrm{~h}$, and then measured every $12 \mathrm{~h}$ until $84 \mathrm{~h}$. NA liquid medium alone was used as a control. At each time point, $\mathrm{OD}_{600}$ was measured by a spectrophotometer at an absorbance wavelength of $600 \mathrm{~nm}$ (DU 800, Beckman Coulter, Brea, CA, USA). For fermentation medium optimization, a liquid medium with glucose $(2.5 \mathrm{~g} / \mathrm{L})$ and peptone $(10 \mathrm{~g} / \mathrm{L})$ as carbon and nitrogen sources, respectively, was used as the basic medium. Then, the glucose carbon source was replaced by either sucrose, lactose, or mannitol, while the peptone nitrogen source was replaced by either beef extract or ammonium nitrate to explore the best carbon and nitrogen source combination for endophyte fermentation. The customization medium was used as a blank control and $\mathrm{OD}_{600}$ was measured after $24 \mathrm{~h}$ of incubation. For fermentation temperature and $\mathrm{pH}$ optimization, the endophyte isolate was cultured at different temperatures $\left(4{ }^{\circ} \mathrm{C}\right.$, $10{ }^{\circ} \mathrm{C}, 16{ }^{\circ} \mathrm{C}, 22{ }^{\circ} \mathrm{C}, 28{ }^{\circ} \mathrm{C}, 34{ }^{\circ} \mathrm{C}, 40{ }^{\circ} \mathrm{C}, 46^{\circ} \mathrm{C}$, and $52{ }^{\circ} \mathrm{C}$ ) and $\mathrm{pH}$ values (3.0, 5.0, 6.0, 7.0, 7.4, 8.0, 10.0, and 12.0) to determine the best growth temperature and $\mathrm{pH}$. The $\mathrm{OD}_{600}$ was measured after $24 \mathrm{~h}$ incubation. The $0.2 \mathrm{M} \mathrm{KOH}$ and $0.2 \mathrm{M} \mathrm{HCl}$ were used to adjust the $\mathrm{pH}$ value of the medium. Each treatment included three replications and two independent experimental duplications.

\subsection{In Vitro Antagonistic Test of Endophyte against F. graminearum}

Testing for the antagonistic effects of endophyte against $F$. graminearum was performed on detached spikelets from the "Zhengmai 9023" cultivar. Detached spikelets were soaked in a suspension of endophyte or control water for $2 \mathrm{~min}$, before being placed on plates filled with water agar medium $(3 \mathrm{~g} / \mathrm{L})$. Four days later, a conidia suspension of $F$. graminearum $\left(10^{5}\right.$ conidia/mL $)$ was sprayed onto spikelets. Then, spikelets were incubated at $20 / 15^{\circ} \mathrm{C}$ day/night temperature with a $16 / 8 \mathrm{~h} \mathrm{light} / \mathrm{dark}$ photoperiod, and $65 \%$ relative humidity. Two weeks later, the disease severity was assessed and scaled to four levels, and level values were used to calculate severity index and protection rate according to methods developed by Comby et al. [8]. Each treatment included at least 15 spikelet replications and two independent experimental duplications. 


\subsection{Statistical Analysis}

Data were subjected to analysis of variance using SPSS 19.0, where F tests were significant at $p<0.05$, and the means were separated by Duncan's Multiple Range test at $p<0.05$.

\section{Results}

\subsection{Identification of Endophytic Bacteria Strain XS-2}

In total, 113 strains of endophytic bacteria were isolated from the roots, stems, leaves, and spikelets tissues of wheat. After testing by dual culture assays, six out of 113 strains showed antagonistic effects on Fusarium graminearum. Among them, XS-2, which is a strain isolated from spikelets, exhibited the best antagonistic effect with a clear inhibition zone being observed between bacterial colonies and fungal mycelium. As shown in Figure 1, the average diameter of the inhibition zone was $29.58 \mathrm{~mm}$, which was significantly larger than the other five strains.
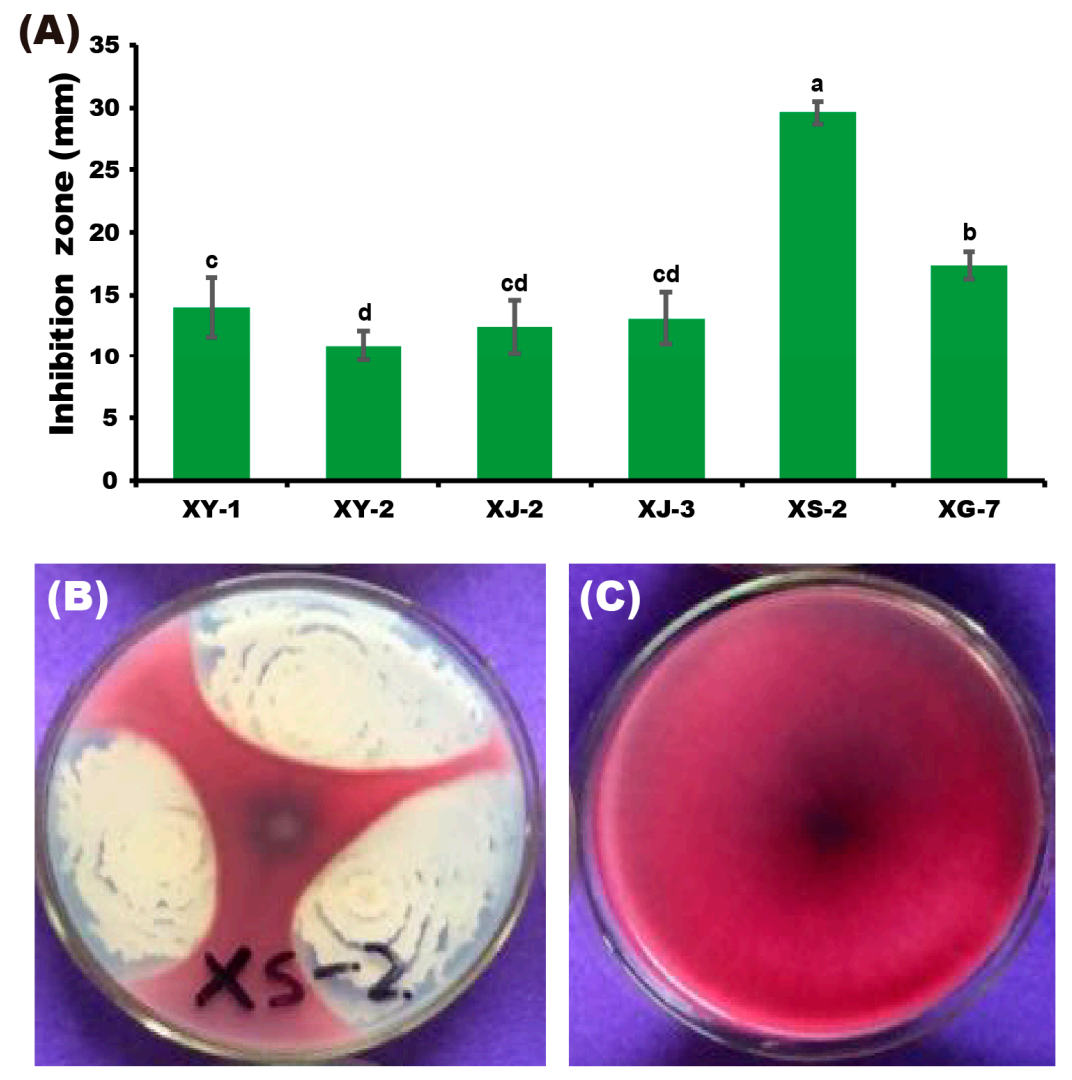

Figure 1. Antagonistic effects of endophytic bacteria on Fusarium graminearum. (A) Inhibition effects of six endophyte isolates against $F$. graminearum. (B) The antagonistic effect of XS-2 against $F$. graminearum and (C) F. graminearum growth alone. Values are means $\pm \mathrm{SD}(n=10)$. Different letters above the bars indicate a significant difference at $p<0.05$.

To further classify this endophyte to a species level, sequence analysis of the 16S rRNA gene was performed. a $1500 \mathrm{bp}$ fragment amplified by universal $16 \mathrm{~S}$ rDNA primers was sequenced. The obtained sequence was aligned with BLAST (Basic Local Alignment Search Tool) on the NCBI (National Center for Biotechnology Information) website (http://www.ncbi.nlm.nih.gov/) to determine the species of strain XS-2. The BLAST result revealed that $16 \mathrm{~S}$ rDNA sequence of XS-2 was highly homologous to sequences belonging to Bacillus species. Then, the $16 \mathrm{~S}$ rDNA sequence of XS-2 and its homologous sequences were collected and used to construct a phylogenetic tree according to the genetic distance. The phylogenetic analysis showed that XS-2 was classified as Bacillus amyloliquefaciens (Figure 2). 


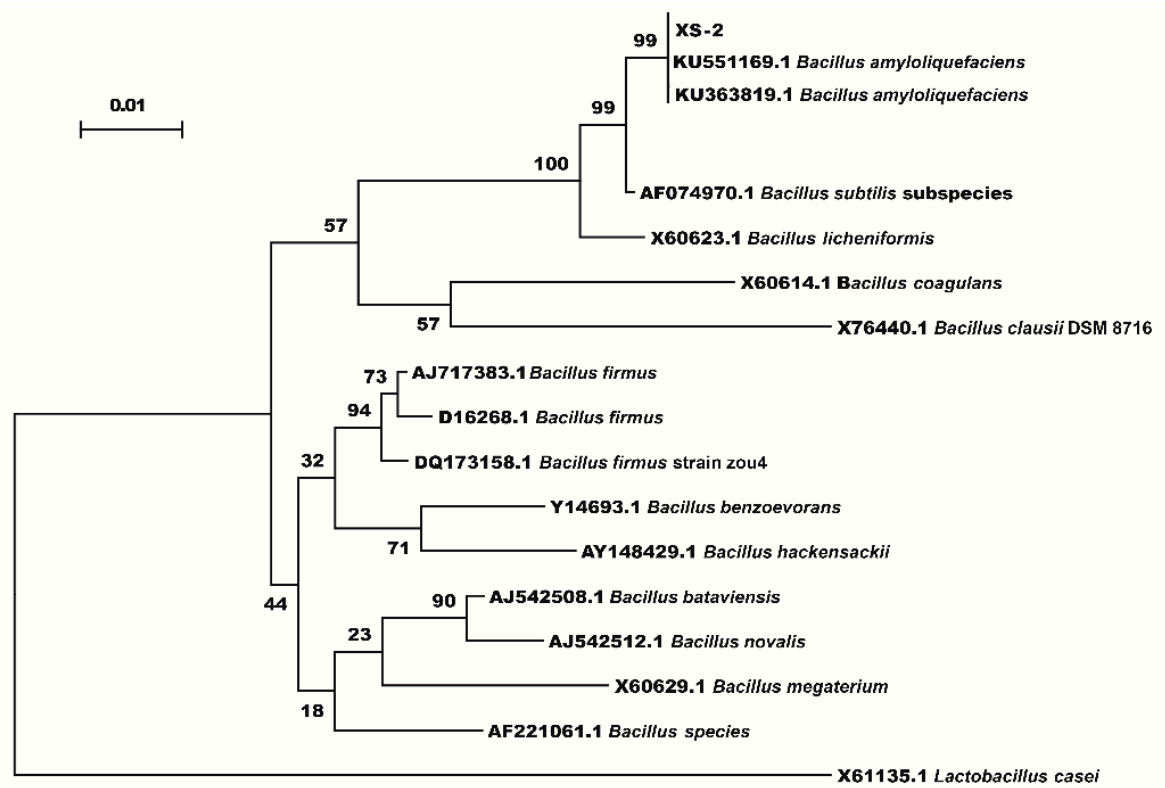

Figure 2. Phylogenetic tree based on the $16 \mathrm{~S}$ rDNA sequence of strain XS-2 and homologous sequences collected from NCBI. The 16S rDNA sequence of XS-2 and homolog sequences from other species were aligned using ClustalW2. The phylogenetic tree was constructed using MEGA7.0 software with the maximum likelihood method (bootstrap values: 1000 replicates). Lactobacillus casei was used as the out-species.

\subsection{Determination of Antimicrobial Spectrum}

In order to test the antimicrobial ability of XS-2, its antagonistic effects against several common crop and fruit tree pathogens, including Fusarium moniliforme and Rhizoctonia solani, Alternaria alternata, Colletotrichum gloeosporioides, Sclerotium rolfsii, and Colletotrichum truncatum, were determined by using dual culturing assays. As shown in Table 1, XS-2 significantly inhibited the growth of all six tested pathogens and the inhibition zones ranged from 18.9 to $33 \mathrm{~mm}$, which implies a broad antimicrobial spectrum of XS-2. In addition, XS-2 showed the greatest inhibitory effect on Alternaria alternata (the pathogen of pear black spot), with the inhibition zone reaching $33 \mathrm{~mm}$.

Table 1. Antagonistic activities of strain XS-2 against different plant pathogens.

\begin{tabular}{cc}
\hline Pathogen & Average Inhibition Zone Diameter (mm) \\
\hline Colletotrichum gloeosporioides & $22.0 \pm 6.8 \mathrm{~d}$ \\
Rhizoctonia solani & $25.4 \pm 6.7 \mathrm{c}$ \\
Sclerotium rolfsii & $29.6 \pm 1.7 \mathrm{~b}$ \\
Alternaria alternata & $33.3 \pm 0.2 \mathrm{a}$ \\
Fusarium moniliforme & $22.3 \pm 0.5 \mathrm{c}, \mathrm{d}$ \\
Colletotrichum truncatum & $23.4 \pm 1.1 \mathrm{c}, \mathrm{d}$ \\
\hline
\end{tabular}

Data are means $\pm \mathrm{SD}(n=3)$. The data were subjected to one-way ANOVA with Duncan's multiple range test using SPSS 19.0 software. Different letters indicate a significant difference among treatments $(p<0.05)$.

\subsection{Determination of the Optimal Fermentation Conditions}

The growth curve of XS-2 was determined by a time series measuring $\mathrm{OD}_{600}$ values. As shown in Figure 3A, the XS-2 strain entered the exponential growth phase for $24 \mathrm{~h}$ after inoculation to NA liquid medium. It was then under the plateau phase for $24-60 \mathrm{~h}$, and entered the decline phase after $60 \mathrm{~h}$ (Figure 3A). 

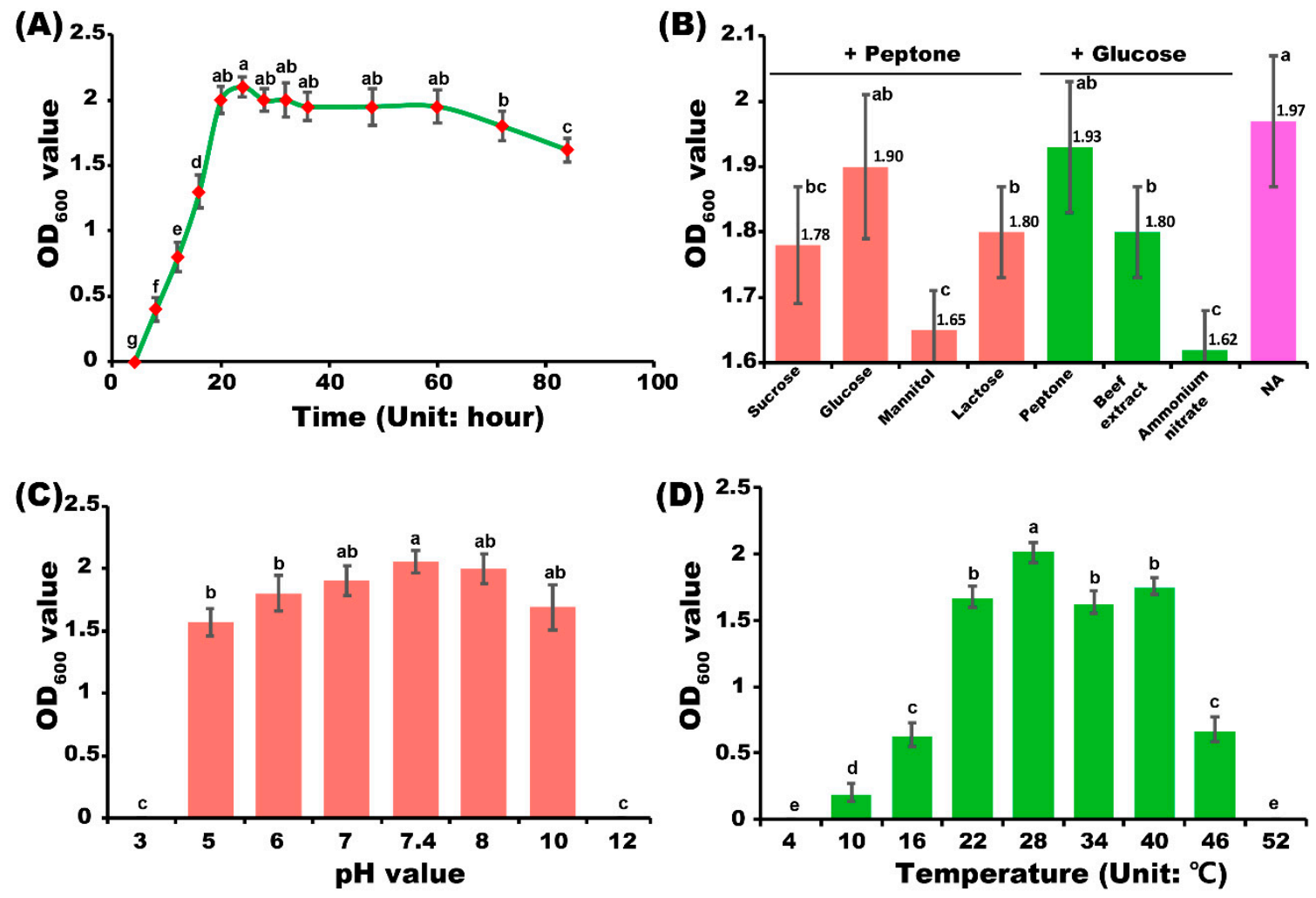

Figure 3. Optimization of fermentation conditions. (A) Growth curve of XS-2 grown in NA medium at different time points $\left(28^{\circ} \mathrm{C}, 180 \mathrm{rpm}\right.$ in dark). (B) Growth of XS-2 in liquid medium with different combinations of carbon and nitrogen sources. (C) Growth of XS-2 in NA medium at different adjusted $\mathrm{pH}$ values. (D) Growth of XS-2 in in NA medium under different temperatures. Each set of conditions received three replications from two independent experimental duplications. Values are means $\pm S D$ $(n=6)$. Different letters above the bars indicate a significant difference at $p<0.05$.

To optimize the fermentation conditions, the influences of different nutrient sources (carbon and nitrogen sources) and growth conditions ( $\mathrm{pH}$ and temperature) on XS-2 were studied. Results showed that all of the carbon and nitrogen sources tested in this study could be utilized by XS-2, with glucose and peptone being the most optimum carbon and nitrogen sources, respectively, since their $\mathrm{OD}_{600}$ values were the highest among all of the growth media (Figure 3B). Considering the fact that glucose and peptone are the two main components of the NA medium, we then directedly tested the fermentation effect of NA medium. As can be seen in Figure 3B, the $\mathrm{OD}_{600}$ value reached 1.97 after $24 \mathrm{~h}$ of incubation, which was higher than any other customized nutrient source combinations. This suggests that NA medium is highly suitable for culturing XS-2.

The bacterial solution of XS-2 was inoculated in NA liquid medium at different $\mathrm{pH}$ levels $\mathrm{pH}=3.0$, 5.0, 6.0, 7.0, 7.4, 8.0, 10.0, and 12.0), and the $\mathrm{OD}_{600}$ values were measured $24 \mathrm{~h}$ later. The results showed that the XS-2 strain can grow in $\mathrm{pH}$ levels ranging from 4.0 to 10.0 , and that the most suitable $\mathrm{pH}$ values for growth ranged from 5.0 to 9.0, with the optimum $\mathrm{pH}$ value being 7.4 (Figure 3C). To determine the optimum temperature, the bacterial liquid of XS-2 was inoculated into NA culture solution (pH adjust to 7.4) and placed in an incubator at different temperatures $\left(4^{\circ} \mathrm{C}, 10{ }^{\circ} \mathrm{C}, 16^{\circ} \mathrm{C}, 22^{\circ} \mathrm{C}, 28{ }^{\circ} \mathrm{C}, 34^{\circ} \mathrm{C}\right.$, $40{ }^{\circ} \mathrm{C}, 46^{\circ} \mathrm{C}$, and $52{ }^{\circ} \mathrm{C}$ ). The results showed that the XS-2 strain could grow from 10 to $46^{\circ} \mathrm{C}$, and that the optimum temperature was $28^{\circ} \mathrm{C}$ (Figure 3D).

\subsection{Antagonistic in Vitro Test of XS-2 on F. graminearum in Detached Spikelets}

The antagonistic effect of XS-2 against $F$. graminearum was further tested in detached wheat spikelets. As shown in Figure 4, F. graminearum produced typical head blight symptoms for two weeks 
after inoculation, while inoculation with XS-2 alone and distilled water did not induce any visible disease symptom (Figure 4). Additionally, XS-2 application largely reduced the disease severity of head blight (Figure 4A,B). The severity index was decreased from 2.37 to 0.94 (Figure 4C). Moreover, the protection rate of XS-2 (60.42\%) was much better than the protection rate of XG-7 (15.72\%) (Figure 4D), in which the antagonistic effect was second to XS-2 in dual culturing assays (Figure 1A).
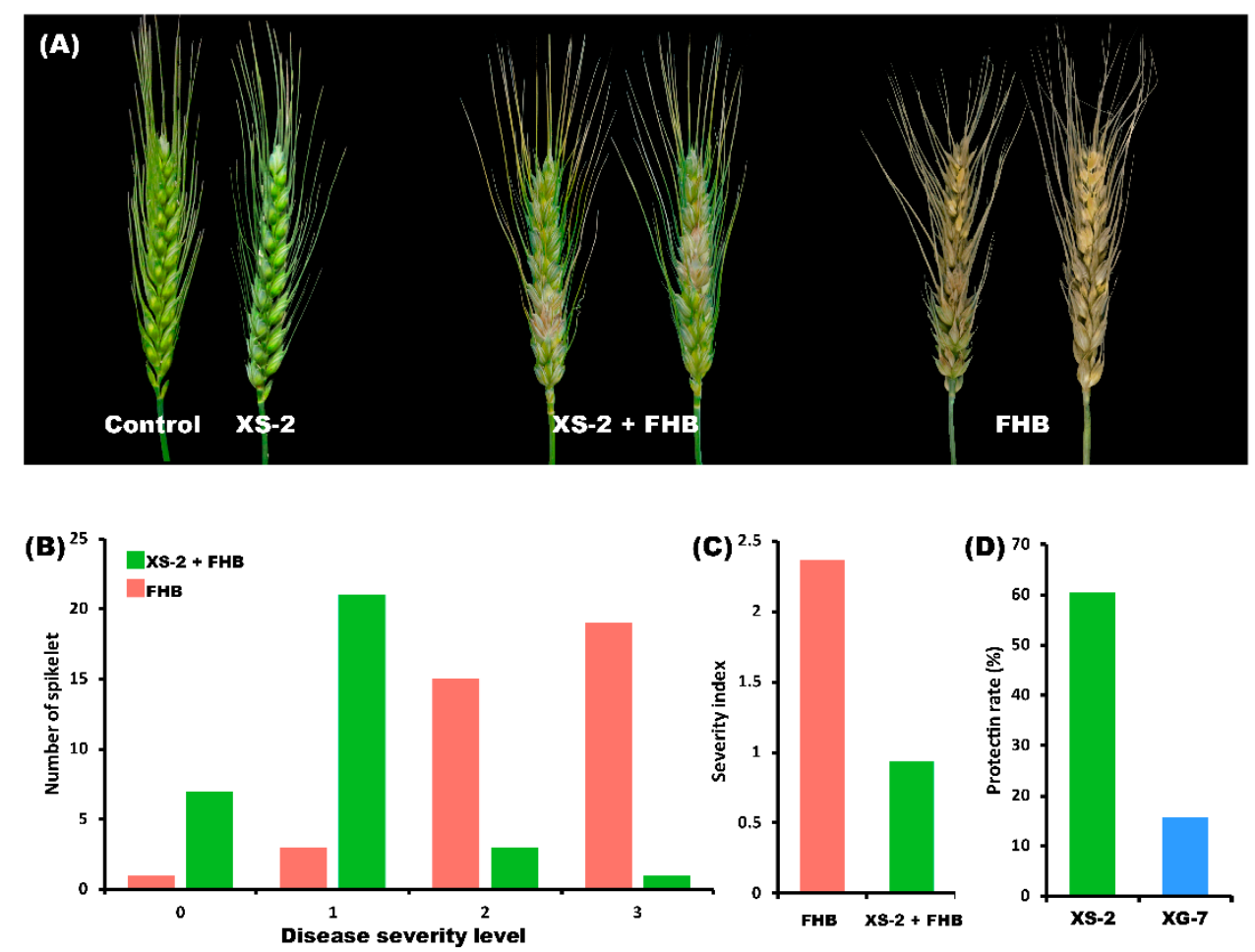

Figure 4. Antagonistic in vitro test of XS-2 against F. graminearum on wheat heads. (A) Symptoms of wheat heads inoculated with XS-2, FHB (Fusarium Head Blight, F. graminearum), and XS-2 plus FHB, distilled water was used as a control. (B) Number of spikelets showing different disease severity levels. (C) The severity index of wheat heads inoculated with XS-2 plus FHB and of wheat heads inoculated with FHB alone. (D) The protection rate of XS-2 compared to XG-7. XG-7 is another endophyte isolated in this study, including the antagonistic effect of which was second to XS-2 in dual culturing assays. At least 32 spikelets from two independent experiments were used. a severity index (SI) was calculated using the following formula: $\left(\mathrm{n}_{0} \times 0+\mathrm{n}_{1} \times 1+\mathrm{n}_{2} \times 2+\mathrm{n}_{3} \times 3\right) / \mathrm{N}$, where $\mathrm{n}_{0}, \mathrm{n}_{1}, \mathrm{n}_{2}$, and $\mathrm{n}_{3}$ represent the number of wheat heads exhibiting disease levels $0,1,2$, and 3 , respectively, and $\mathrm{N}$ represents the total number of wheat heads used. The protection rate was calculated by the following formula: $\left(\mathrm{SI}_{\mathrm{FHB}}\right.$ $\left.-\mathrm{SI}_{(\mathrm{XS}-2}+\mathrm{FHB}\right) / \mathrm{SI}_{\mathrm{FHB}} \times 100$, where $\left.\mathrm{SI}_{(\mathrm{XS}-2}+\mathrm{FHB}\right)$ and $\mathrm{SI}_{\mathrm{FHB}}$ represent the SI values of the XS-2 plus FHB treatment and the FHB treatment alone, respectively. This is similar to the protection rate of XG-7.

\section{Discussion}

In recent years, the wheat Fusarium head blight (FHB) epidemic has become more serious. Due to the shortage of resistant varieties, the control of wheat scab is highly dependent on chemical pesticides. However, with their excessive usage, resistance of Fusarium graminearum to chemical pesticides is increasing, which results in many fungicides losing their efficacy $[5,6]$. Moreover, due to difficulty in accurately predicting the infection period of wheat spike, farmers often miss the optimal pesticide control period $[17,18]$. Therefore, the development of new strategies for wheat FHB control is urgently required.

In this study, we isolated one endophytic bacteria strain XS-2 from spikelets, which exhibited antagonistic effects on F. graminearum, as well as on crop and fruit tree pathogens including F. moniliforme and Rhizoctonia solani (two cotton pathogens), Sclerotium rolfsii (a rice pathogen), 
Alternaria alternata (a pear fruit pathogen), Colletotrichum truncatum (a pitaya fruit pathogen), and Colletotrichum gloeosporioides (an apple fruit pathogen) (Figure 4, Table 1). Specifically, XS-2 had the best inhibitory effect against $A$. alternata. Therefore, it seems that XS-2 exerted a wide antagonistic spectrum and that it could be developed as a potential biocontrol agent to control plant diseases, especially for F. moniliforme and A. alternata.

Through 16S rDNA sequence analysis, the antagonistic bacteria XS-2 isolated from wheat spikelets in this experiment was identified as Bacillus amyloliquefaciens. The B. amyloliquefaciens strains have been reported to possess good biocontrol effects against many plant pathogens. For example, the FZB42 strain has been reported to suppress disease and promote the growth of many plant species, including tomato, cucumber, cotton, tobacco, and lettuce [19]. Strain PPCB004 has been shown to have an antagonistic effect on Botrytis cinerea, Penicillium expansum, and Rhizopus stolonifer in peach fruits, and could be used as a potential biocontrol agent for fruit post-harvest disease management [20]. Similarly, the results of the present study also support that the B. amyloliquefaciens has an antagonistic effect against several crop and fruit tree diseases, which makes it a valuable resource for developing biocontrol agents.

Some studies have suggested that Bacillus species are root dwelling bacteria [19], but, in this study, the species was isolated from wheat spikelets and showed the ability to suppress the development of head blight in spikelets. Coincidentally, Yoshida et al. [21] isolated a B. amyloliquefaciens strain, RC-2, from mulberry leaves, which inhibited the growth of several phytopathogenic fungi and bacteria. These results confirm the fact that $B$. amyloliquefaciens strains existed in non-root tissues including leaves and spikelets. However, it is still unclear whether those B. amyloliquefaciens isolated from roots possess similar characteristics (e.g., antimicrobial spectrum) to those isolated from other tissues. Moreover, the subspecific taxonomic status of XS-2 is still unclear, which is necessary for comparisons with other strains [22]. Further work is needed to explore the subspecific taxonomic status of B. amyloliquefaciens strain XS-2 isolated in our study.

To optimize the fermentation conditions of XS-2, different cultivation conditions, including nutrient sources, $\mathrm{pH}$, and temperature, were analyzed. The results showed that canonical NA medium (10 $\mathrm{g}$ of peptone, $3 \mathrm{~g}$ of beef extract, and $2.5 \mathrm{~g}$ of glucose per $\mathrm{L}$ ) is highly suitable for the cultivation of XS-2 (Figure 3B), and that the optimal $\mathrm{pH}$ and temperature were 7.4 and $28^{\circ} \mathrm{C}$, respectively (Figure 3C,D). a wide range of temperatures $\left(4-52{ }^{\circ} \mathrm{C}\right)$ were used during the experiment. This is because wheat experiences a large range of temperatures during its growth period from a subzero temperature in the winter to occasionally more than $40^{\circ} \mathrm{C}$ in the summer [23,24]. Additionally, testing a wide range of fermentation temperatures provides a theoretical basis for the application of XS-2 under different temperatures in the field.

To further confirm the antagonistic effects of XS-2 on wheat FHB disease, we then performed an in vitro test on wheat heads under laboratory conditions (Figure 4). The results showed that XS-2 application largely reduced the disease severity of FHB, since the disease severity index was decreased from 2.37 to 0.94 . This is strong evidence of the biocontrol potential of XS-2 on FHB disease. In addition, we also compared the antagonistic effects of XS-2 to that of XG-7 (another endophytic bacterium isolated in this study, which exhibited an antagonistic effect second to XS-2 in dual culturing assays). The results suggested that XS-2 had a much greater antagonistic effect than XG-7, since the protection rate of XS-2 (60.42\%) was four times greater than XG-7 (15.72\%) (Figure 4D). However, we must emphasize that a short period of pathogen exposure to XS-2 in culture medium or in wheat head inoculated under laboratory conditions may not represent the actual scenario for plants that are subjected to long-term stress in field conditions. Therefore, more work is needed to test the antagonistic effects of XS-2 in the field and to optimize the field usage strategies including field application time, spray concentration, and a proper addition agent $[25,26]$. Nevertheless, our study provides theoretical support for the practical application of XS-2 as biocontrol bacteria in crop production, which is environmentally-friendly and pollution-free.

Author Contributions: Conceptualization, D.M. and P.Z. Methodology, D.M., L.Y., and H.Y. Software, J.Y., W.X., and J.Z. Validation, D.M., J.Y., and L.Y. Formal analysis, P.Z. Investigation, P.Z. Resources, D.M. Data curation, P.Z. 
Writing—original draft preparation, Y.Z. and P.Z. Writing—review and editing, Y.Z., P.Z., and J.Y. Visualization, P.Z. Supervision, J.Y. and D.M. Project administration, D.M. Funding acquisition, J.Y. and D.M.

Funding: The "National Key Research and Development Foundation, \#2018YFD020050602 and \#2017YFD01008002," "Engineering Research Center of Ecology and Agricultural Use of Wetland, Ministry of Education, \#KF201909," and "National Key Laboratory of Diseases and Insect Biology Open Found, \#SKLOF201707" funded this research.

Acknowledgments: We thank Yongli Qiao for beneficial comments on the initial project design and data analysis.

Conflicts of Interest: The authors declare no conflict of interest.

\section{References}

1. Jung, B.; Park, S.-Y.; Lee, Y.-W.; Lee, J. Biological Efficacy of Streptomyces sp. Strain BN1 against the Cereal Head Blight Pathogen Fusarium graminearum. Plant Pathol. J. 2013, 29, 52-58. [CrossRef]

2. Crane, J.M.; Gibson, D.M.; Vaughan, R.H.; Bergstrom, G.C. Iturin Levels on Wheat Spikes Linked to Biological Control of Fusarium Head Blight by Bacillus amyloliquefaciens. Phytopathology 2013, 103, 146-155. [CrossRef]

3. Draeger, R.; Gosman, N.; Steed, A.; Chandler, E.; Thomsett, M.; Schondelmaier, J.; Buerstmayr, H.; Lemmens, M.; Schmolke, M.; Mesterhazy, A.; et al. Identification of QTLs for resistance to Fusarium head blight, DON accumulation and associated traits in the winter wheat variety Arina. Theor. Appl. Genet. 2007, 115, 617-625. [CrossRef]

4. Miu, W.H. Characteristics and high yield cultivation of Sheng selected No. 6 wheat. Bull. Agric. Sci. Technol. 2010, 7, 139-140.

5. Yin, J.L.; Zhang, X.; Ma, D.F.; Lu, C.; He, Y.Q.; Zhu, Y.X. a study on the sensitivity and synergistic effect of prochloraz and cypermethrin on Gibberella zeae in wheat. Acta Agric. Univ. Jiangxiensis 2018, 40, 920-924.

6. Xia, Y.C.; Zhu, Y.X.; Ma, D.F.; Liu, L.C.; Yin, J.L. Isolation and identification of antagonistic strain of Fusarium graminearum. Acta Agric. Univ. Jiangxiensis 2018, 41, 33-42.

7. Khan, N.I.; Schisler, D.A.; Boehm, M.J.; Slininger, P.J.; Bothast, R.J. Selection and Evaluation of Microorganisms for Biocontrol of Fusarium Head Blight of Wheat Incited by Gibberella zeae. Plant Dis. 2001, 85, 1253-1258. [CrossRef]

8. Comby, M.; Gacoin, M.; Robineau, M.; Rabenoelina, F.; Ptas, S.; Dupont, J.; Profizi, C.; Baillieul, F. Screening of wheat endophytes as biological control agents against Fusarium head blight using two different in vitro tests. Microbiol. Res. 2017, 202, 11-20. [CrossRef]

9. Shahzad, R.; Khan, A.L.; Bilal, S.; Waqas, M.; Kang, S.-M.; Lee, I.-J. Inoculation of abscisic acid-producing endophytic bacteria enhances salinity stress tolerance in Oryza sativa. Environ. Exp. Bot. 2017, 136, 68-77. [CrossRef]

10. Mota, M.S.; Gomes, C.B.; Souza Júnior, I.T.; Moura, A.B. Bacterial selection for biological control of plant disease: Criterion determination and validation. Braz. J. Microbiol. 2017, 48, 62-70. [CrossRef]

11. Bello, G.D.; Mónaco, C.; Simon, M.R. Biological control of seedling blight of wheat caused by Fusarium graminearum with beneficial rhizosphere microorganisms. World J. Microbiol. Biotechnol. 2002, 18, 627-636. [CrossRef]

12. Alimi, M. Characterization and application of microbial antagonists for control of Fusarium head blight of wheat caused by Fusarium graminearum using single and mixture strain of antagonistic bacteria on resistance and susceptible cultivars. Afr. J. Microbiol. Res. 2012, 6, 326-334.

13. Palazzini, J.M.; Alberione, E.; Torres, A.; Donat, C.; Köhl, J.; Chulze, S. Biological control of Fusarium graminearum sensu stricto, causal agent of Fusarium head blight of wheat, using formulated antagonists under field conditions in Argentina. Boil. Control 2016, 94, 56-61. [CrossRef]

14. Dimkpa, C.O.; McLean, J.E.; Britt, D.W.; Anderson, A.J. Antifungal activity of ZnO nanoparticles and their interactive effect with a biocontrol bacterium on growth antagonism of the plant pathogen Fusarium graminearum. BioMetals 2013, 26, 913-924. [CrossRef]

15. Liu, H.F.; Zhou, J.; Liao, J.; Yi, J.P.; Ma, D.F.; Deng, J.X. Grafted twig rot on Citrus sinensis caused by a member of the Fusarium solani species complex. Can. J. Plant Pathol. 2019, 1-7. [CrossRef]

16. Kumar, S.; Stecher, G.; Tamura, K. MEGA7: Molecular Evolutionary Genetics Analysis version 7.0 for bigger datasets. Mol. Boil. Evol. 2016, 33, 1870-1874. [CrossRef] 
17. Malandrakis, A.A.; Markoglou, A.N.; Konstantinou, S.; Doukas, E.G.; Kalampokis, J.F.; Karaoglanidis, G.S. Molecular characterization, fitness and mycotoxin production of benzimidazole-resistant isolates of Penicillium expansum. Int. J. Food Microbiol. 2013, 162, 237-244. [CrossRef]

18. Xu, J.H.; Wang, J.W.; Hu, X.D.; Ji, F.; Shi, J.R. Isolation, identification of antagonistic bacteria AF0907 against Fusarium graminearum and its characteristics. Jiangsu J. Agric. Sci. 2013, 29, 517-522.

19. Chowdhury, S.P.; Hartmann, A.; Gao, X.; Borriss, R. Biocontrol mechanism by root-associated Bacillus amyloliquefaciens FZB42-A review. Front. Microbiol. 2015, 6, 780. [CrossRef]

20. Arrebola, E.; Sivakumar, D.; Bacigalupo, R.; Korsten, L. Combined application of antagonist Bacillus amyloliquefaciens and essential oils for the control of peach postharvest diseases. Crop. Prot. 2010, 29, 369-377. [CrossRef]

21. Yoshida, S.; Hiradate, S.; Tsukamoto, T.; Hatakeda, K.; Shirata, A. Antimicrobial Activity of Culture Filtrate ofBacillus amyloliquefaciensRC-2 Isolated from Mulberry Leaves. Phytopathology 2001, 91, 181-187. [CrossRef]

22. Fan, B.; Blom, J.; Klenk, H.-P.; Borriss, R. Bacillus amyloliquefaciens, Bacillus velezensis, and Bacillus siamensis Form an "Operational Group B. amyloliquefaciens" within the B. subtilis Species Complex. Front. Microbiol. 2017, 8, 1807. [CrossRef]

23. Porter, J.R.; Gawith, M. Temperatures and the growth and development of wheat: a review. Eur. J. Agron. 1999, 10, 23-36. [CrossRef]

24. Asseng, S.; Foster, I.; Turner, N.C. The impact of temperature variability on wheat yields. Glob. Chang. Boil. 2011, 17, 997-1012. [CrossRef]

25. Collins, D.P.; Jacobsen, B.J. Optimizing a Bacillus subtilis isolate for biological control of sugar beet cercospora leaf spot. Boil. Control 2003, 26, 153-161. [CrossRef]

26. Chiou, A.L.; Wu, W.S. Formulation of Bacillus amyloliquefaciens B190 for Control of Lily Grey Mould (Botrytis elliptica). J. Phytopathol. 2003, 151, 13-18. [CrossRef]

(C) 2019 by the authors. Licensee MDPI, Basel, Switzerland. This article is an open access article distributed under the terms and conditions of the Creative Commons Attribution (CC BY) license (http://creativecommons.org/licenses/by/4.0/). 